\title{
Pesquisa em terapia ocupacional: apontamentos acerca dos caminhos acadêmicos no cenário nacional*
}

\section{Research in occupational therapy: notes about the academics ways in the national scene}

\author{
Roseli Esquerdo Lopes ${ }^{1}$, Ana Paula Serrata Malfitano² Fátima \\ Corrêa Oliver ${ }^{3}$, Sara Caram Sfair ${ }^{4}$, Thaís Juliana Medeiros ${ }^{5}$
}

\begin{abstract}
LOPES, R. E.; MALFITANO, A. P. S.; OLIVER, F. C.; SFAIR, S. C.; MEDEIROS, T. J. Pesquisa em terapia ocupacional: apontamentos acerca dos caminhos acadêmicos no cenário nacional. Rev. Ter. Ocup. Univ. São Paulo, v. 21, n. 3, p. 207-214, set./dez. 2010.
\end{abstract}

\begin{abstract}
RESUMO: A área de terapia ocupacional vem discutindo, em seus fóruns coletivos, estratégias de organização para acesso a financiamentos de pesquisa e sua institucionalização no âmbito da pós-graduação stricto sensu. Nesse cenário, apresentam-se aqui dados referentes aos Grupos de Pesquisa em Terapia Ocupacional no país. Foram levantados, no Diretório de Grupos de Pesquisa do $\mathrm{CNPq}$ (grande área da Saúde, subárea de Fisioterapia e Terapia Ocupacional), os grupos registrados com o termo "Terapia Ocupacional" no nome, no título de linha de pesquisa ou em suas palavraschave. Posteriormente, foram contatados todos os líderes dos grupos, solicitando-lhes dados sobre as pesquisas em andamento, os pesquisadores integrantes, bem como os financiamentos acessados. Foram encontrados 26 grupos ativos, com grande concentração na região Sudeste do país e com um universo de 172 pesquisadores, sendo 91 doutores. Foram declarados 124 projetos de pesquisa em andamento, sendo que 65 receberam algum tipo de financiamento. Verifica-se que a área possui um número ainda pequeno de doutores, com baixa inserção em programas de pós-graduação stricto sensu, elementos considerados relevantes para o desenvolvimento da pesquisa específica; ainda, que precisa lidar com o desafio de atingir patamares representativos, tendo, portanto, que crescer junto às instituições que certificam a produção do conhecimento acadêmico.
\end{abstract}

DESCRITORES: Pesquisa; Financiamento da pesquisa; Diretórios de pesquisadores; Terapia ocupacional/tendências; Educação superior.

\footnotetext{
* Parte deste texto foi apresentada no XII Encontro Nacional de Docentes em Terapia Ocupacional, ocorrido em Curitiba, na Universidade Federal do Paraná, em setembro de 2010.

1. Professora Associada do Departamento de Terapia Ocupacional e dos Programas de Pós-Graduação em Educação e em Terapia Ocupacional da Universidade Federal de São Carlos (UFSCar).

2. Professora Adjunta do Departamento de Terapia Ocupacional e do Programa de Pós-Graduação em Terapia Ocupacional da UFSCar.

3. Professora Doutora do Departamento de Fisioterapia, Fonoaudiologia e Terapia Ocupacional e do Programa de Pós-Graduação em Ciências da Reabilitação da Faculdade de Medicina da Universidade de São Paulo.

4. Terapeuta Ocupacional pela UFSCar. Mestranda do Programa de Pós-Graduação em Terapia Ocupacional da UFSCar.

5. Cientista Social pela UFSCar. Mestranda do Programa de Pós-Graduação em Terapia Ocupacional da UFSCar.
}

Endereço para correspondência: Universidade Federal de São Carlos. Departamento de Terapia Ocupacional - Laboratório METUIA. Rodovia Washington Luís, km. 235, São Carlos-SP. CEP: 13565-905. E-mail: relopes@ufscar.br 


\section{INTRODUÇÃO}

$\mathrm{O}$ s dados aqui apresentados são fruto da organização para desenvolvimento do Grupo de Trabalho: "Desafios e estratégias para acesso ao financiamento de pesquisa em terapia ocupacional", realizado durante o XII Encontro Nacional de Docentes em Terapia Ocupacional, ocorrido em Curitiba, PR, em 2010. Tais discussões foram propostas em continuidade aos trabalhos iniciados em 2008, na Universidade de São Paulo, durante o XI Encontro Nacional de Docentes de Terapia Ocupacional (LOPES et al., 2008), e no I Seminário Nacional de Pesquisa em Terapia Ocupacional, ocorrido em 2009, na Universidade Federal de São Carlos.

Essas articulações demonstram a preocupação da área com sua representatividade e crescimento nas instituições que certificam a produção do conhecimento acadêmico. Para contribuir com dados para essa discussão, bem como para disponibilizar elementos para análises acerca do panorama da área de terapia ocupacional no âmbito da pesquisa e no diálogo com as agências de fomento, trabalhou-se a partir de um levantamento de dados referentes aos Grupos de Pesquisa, registrados no Diretório de Grupos de Pesquisa do CNPq (Conselho Nacional de Desenvolvimento Científico e Tecnológico). Esse levantamento partiu do termo "Terapia Ocupacional" no nome do grupo, no título de linha de pesquisa ou em suas palavras-chave, em grupos que integram a chamada grande área da Saúde, subárea de Fisioterapia e Terapia Ocupacional. Posteriormente, contatou-se, por telefone e/ou endereço eletrônico, todos os líderes dos grupos identificados, quando foram solicitados dados referentes aos pesquisadores integrantes, às pesquisas em andamento, bem como aos financiamentos acessados.

\section{SÍNTESE DE UMA HISTÓRIA RECENTE: DISCUS- SÕES COLETIVAS ACERCA DA PESQUISA EM TERAPIA OCUPACIONAL}

Desde 2008, momento em que a pauta da pesquisa alcançou mais significativamente os debates coletivos organizados da área, traça-se um perfil da singular situação do campo da terapia ocupacional no universo da produção e divulgação do conhecimento, com ênfase na questão da dificuldade de sua expansão e institucionalização no sistema brasileiro, como também na necessidade de uma organização coletiva para o enfrentamento desse quadro.

As demandas para a realização de pesquisas e produção científica em terapia ocupacional têm sido crescentes no cenário brasileiro. Isso se deve, em parte, ao crescimento de vagas públicas no ensino superior, especialmente no sistema federal, na última década ( 78 cursos de graduação autorizados para funcionamento, segundo dados de 2010 do Instituto Nacional de Estudos e Pesquisas Educacionais Anísio Teixeira - INEP), e à existência de 3 programas de pós-graduação stricto sensu integrando a denominada Área 21 da Coordenação de Aperfeiçoamento de Pessoal de Nível Superior - CAPES, que agrega programas de mestrado acadêmico, mestrado profissional e doutorado nas áreas de Educação Física, Fonoaudiologia, Fisioterapia e Terapia Ocupacional.

O XI Encontro Nacional de Docentes em Terapia Ocupacional, de 2008, discutiu a situação marginal da área no contexto do sistema de pós-graduação brasileiro, o que tem dificultado seu desenvolvimento e crescimento. Tal cenário advém do fato de poucos pesquisadores do campo da terapia ocupacional estarem vinculados como orientadores a programas de mestrado ou doutorado ${ }^{2}$ e, dentre estes, a apenas 3 serem programas da Área 21 -CAPES e a somente 1 ser um programa específico, e recente, de terapia ocupacional. Além disso, a divulgação do conhecimento produzido por pesquisadores terapeutas ocupacionais se concentra em revistas brasileiras classificadas pela Área 21 - CAPES como de baixos índices de impacto, o que tem dificultado o atendimento aos critérios estabelecidos para ingresso pleno na pós-graduação. Isso dificulta tanto o credenciamento de terapeutas ocupacionais nos programas existentes como a criação de novos programas de pós-graduação, com prejuízo para o processo de formação de mestres e doutores na área e para o desenvolvimento e a consolidação de linhas específicas de pesquisa.

É importante ressaltar que as pesquisas e seu financiamento são a matriz de artigos e publicações. Ou seja, há um círculo vicioso perverso e restritivo ao nosso desenvolvimento: poucos projetos financiados/baixa produtividade de artigos/baixa produção na área/baixo número de projetos financiados. Outro aspecto decorrente, é que essas exigências nos levam a não fomentarmos nossos próprios periódicos, o que retroalimenta essa situação (LOPES et al., 2008).

1.Grupo de Trabalho da Rede Nacional de Ensino em Terapia Ocupacional, coordenado pelas professoras Roseli Esquerdo Lopes, Ana Paula Serrata Malfitano e Fátima Côrrea Oliver e que teve uma sessão no XII Encontro Nacional de Docentes em Terapia Ocupacional. 2. Até outubro de 2009, havia 19 pesquisadores vinculados como orientadores de programas de mestrado e 7 como orientadores em programas de doutorado (OLIVER, 2009). 
Uma das propostas deliberadas no XI Encontro de Docentes foi a realização de um Seminário Nacional de Pesquisa, o que se efetivou em novembro de 2009. Foi decidido, igualmente, o encaminhamento ao CNPq da solicitação de uma representação própria da Terapia Ocupacional no Comitê Multidisciplinar em Saúde e a discussão de critérios de proporcionalidade na distribuição de recursos com equiparação de oportunidades entre as áreas. Esse posicionamento foi registrado pelo envio de correspondência específica do evento e da Rede Nacional de Ensino em Terapia ocupacional - RENETO para a Coordenação do Comitê de Assessoramento da Área de Educação Física, Fonoaudiologia, Fisioterapia e Terapia Ocupacional do CNPq (Comitê Multidisciplinar em Saúde), para a Coordenação do Programa de Pesquisa em Saúde do CNPq e para os Representantes da Área de Fisioterapia e Terapia Ocupacional junto ao Comitê de Assessoramento do CNPq. Numa direção mais interna aos pesquisadores da área, discutiu-se e indicou-se a necessidade de se aumentar a demanda de solicitações de financiamento de projetos específicos junto ao CNPq, nos seus diversos editais, notadamente no Edital Universal. Formulou-se e aprovou-se a proposta de que cada grupo de pesquisa da área submetesse pelo menos dois projetos ao próximo Edital Universal (2009) Abordou-se, ainda, a necessidade de conhecimento do teor dos pareceres elaborados pelos assessores do Comitê Multidisciplinar em Saúde, uma vez que não é prática do $\mathrm{CNPq}$ o envio dos mesmos aos proponentes, dificultando o processo de aprimoramento dos projetos de pesquisa apresentados. Por fim, frente à possibilidade de um edital específico no Comitê Multidisciplinar do CNPq, discutiu-se acerca da importância de que este fosse o mais amplo possível para que pudesse contemplar diferentes abordagens em terapia ocupacional. Fruto de intenso trabalho dos integrantes do Comitê Multidisciplinar em Saúde, o CNPq publicou o Edital MCT/ CNPq/CT-Saúde № 58/2009 - "Envelhecimento, Trabalho e Saúde: Promoção da qualidade de vida da população brasileira por meio de atividade física, ocupacional e linguagem", no qual foram contempladas sete propostas, nenhuma delas liderada por pesquisador terapeuta ocupacional.

No final de 2009, realizou-se o I Seminário Nacional de Pesquisa em Terapia Ocupacional, que contou com a participação de 127 pessoas, dentre elas pesquisadores, docentes, terapeutas ocupacionais e alunos da graduação em terapia ocupacional, indo além das expectativas da organização. Constituiu-se como um momento diferenciado para se abrir canais específicos de conhecimento e debate acerca dos critérios vigentes de avaliação dos pesquisadores e dos seus projetos, um espaço público de encontro entre pesquisadores e representantes das principais agências de fomento brasileiras em diálogo direto com a terapia ocupacional. Reconheceu-se, assim, que os critérios vigentes são contestáveis para uma área com pouco acúmulo de história na pesquisa, como a terapia ocupacional. Todavia, são critérios convencionados, o que demanda uma postura de luta dos pesquisadores terapeutas ocupacionais com vistas ao tensionamento qualificado dos processos avaliativos e da inserção de especificidades da terapia ocupacional, sem que se descuide da qualidade acadêmica das proposições (LOPES et al., 2009).

Os encaminhamentos do Seminário centraram-se sobre: - a necessidade de articulação entre as proposições de ensino da graduação, extensão e pesquisa; - a necessidade de maior apropriação por parte dos pesquisadores da área dos códigos das agências de fomento à pesquisa e de seus critérios; - o fortalecimento dos periódicos específicos da área de terapia ocupacional; - a constituição de parcerias intrainstitucionais e interinstitucionais (nacionais e internacionais), por meio virtual e/ou presencial; - a realização de oficinas e/ou workshops de elaboração de artigos científicos; - a continuidade de espaços para a discussão específica da pesquisa nos eventos da área; e, finalmente, - a realização do II Seminário Nacional de Pesquisa em Terapia Ocupacional até 2011 (LOPES; MALFITANO, 2009).

Em 2010, no XII Encontro Nacional de Docentes de Terapia Ocupacional, o Grupo de Trabalho "Desafios e estratégias para o acesso ao financiamento de pesquisas em Terapia Ocupacional", conforme estabelecido em 2008, foi mantido na programação, com os objetivos de - divulgar e debater o perfil de acesso a financiamentos e apoios à pesquisa em terapia ocupacional junto a agências de fomento nacionais e regionais; - debater e indicar estratégias de curto e médio prazos para o desenvolvimento de pesquisa em terapia ocupacional. Aprofundou-se a discussão sobre as mesmas temáticas em tela nos encontros anteriores, reafirmando-se e deliberando-se encaminhamentos semelhantes, com vistas ao avanço da área no que tange ao contexto da pesquisa.

\section{GRUPOS DE PESQUISA EM TERAPIA OCUPACIO- NAL NO PAIIS: PERFIL E FINANCIAMENTO}

Em setembro de 2010, o levantamento de Grupos de Pesquisa cadastrados no Diretório de Grupos de Pesquisa do CNPq, na grande área da Saúde, subárea de Fisioterapia e Terapia Ocupacional, que apresentassem o termo "Terapia Ocupacional" no nome do grupo, no título de linha de pesquisa ou em suas palavras-chave encontrou 30 grupos registrados, sendo que 3 deles não apresentavam em sua composição terapeutas ocupacionais e suas linhas de pes- 
quisa não eram específicas da área ${ }^{3}$. Havia, ainda, um grupo inativo e dois grupos não atualizados há mais de doze meses. Sendo assim, não se contabilizou os três grupos que não contavam com a participação de terapeutas ocupacionais e o grupo inativo, trabalhando-se com um universo de 26 grupos da área, apresentados na Tabela 1.

Tabela 1 - Grupos de Pesquisa da Área de Terapia Ocupacional Cadastrados no CNPq,segundo Instituições de Ensino Superior

\begin{tabular}{|c|c|c|}
\hline \multicolumn{2}{|c|}{ Nomes dos Grupos de Pesquisa } & \multirow{2}{*}{$\begin{array}{l}\text { Instituição*** } \\
\text { IFRJ }\end{array}$} \\
\hline 1 & Inclusão, Corpo, Arte e Transdisciplinaridade (InCArT) & \\
\hline 2 & Saúde Mental, Terapia Ocupacional e Perspectivas de Formação & PUC-Campinas \\
\hline 3 & Avaliação do desenvolvimento e desempenho infantil & UFMG \\
\hline 4 & Estudos dos processos de funcionalidade e de incapacidade relacionados ao desenvolvimento humano & UFMG \\
\hline 5 & Laboratório de estudos em Ciência da Ocupação & UFPA \\
\hline 6 & Fundamentos e Clínica da Terapia Ocupacional & UFPE \\
\hline 7 & Modos de Vida e Territórios Urbanos & UFPE \\
\hline 8 & Grupo de Pesquisa em Terapia Ocupacional & UFPR \\
\hline 9 & Estudos em Reabilitação Física, Tecnologia Assistiva e Funcionalidade & UFSCar \\
\hline 10 & Formação e Capacitação em Terapia Ocupacional & UFSCar \\
\hline 11 & Terapia Ocupacional e Educação no Campo Social & UFSCar \\
\hline 12 & Terapia ocupacional e Gerontologia & UFSCar \\
\hline 13 & Terapia Ocupacional e Saúde Mental & UFSCar \\
\hline 14 & Terapia Ocupacional: Atividade, tecnologias, desenvolvimento e qualidade de vida. & UFSCar \\
\hline 15 & Saúde e Cidadania: Processos de vulnerabilidades e possibilidades de intervenção no ciclo de vida & UFTM \\
\hline 16 & Grupo de Estudos e Pesquisas em Trabalho e Saúde & UNIFESP \\
\hline 17 & Laboratório de Estudos e Pesquisa da Enação & UNIFESP \\
\hline 18 & $\begin{array}{l}\text { Atividade, Cotidiano e Cuidado: Terapia Ocupacional e os Processos de Adoecimento, Hospitalização e } \\
\text { Vulnerabilização na Infância e Adolescência }\end{array}$ & $\mathrm{USP} / \mathrm{SP}$ \\
\hline 19 & Grupo de Estudo de Trabalho, Saúde e Terapia Ocupacional & USP/SP \\
\hline 20 & Laboratório de Estudo e Pesquisa Arte e Corpo em Terapia Ocupacional & $\mathrm{USP} / \mathrm{SP}$ \\
\hline 21 & Laboratório de Estudos sobre Deficiência e Cotidiano & USP/SP \\
\hline 22 & Laboratório de Investigação sobre a atividade humana & USP/RP \\
\hline 23 & Laboratório de Pesquisa em Inovação e Tecnologia Assistiva - LAPITEC & USP/RP \\
\hline 24 & LEPTOI - Laboratório de Ensino e Pesquisa em Terapia Ocupacional, Infância e Adolescência & USP/RP \\
\hline 25 & Políticas, ações sociais, cultura e reabilitação & USP/SP \\
\hline 26 & REATA - Laboratório de Estudos em Reabilitação e Tecnologia Assistiva & USP/SP \\
\hline
\end{tabular}

Fonte: $\mathrm{CNPq}(2010)$

** IFRJ: Instituto Federal de Rio de Janeiro; PUC-Campinas: Pontifícia Universidade Católica de Campinas; UFMG: Universidade Federal de Minas Gerais; UFPA: Universidade Federal do Pará; UFPE: Universidade Federal de Pernambuco; UFPR: Universidade Federal do Paraná; UFSCar: Universidade Federal de São Carlos; UFTM: Universidade Federal do Triângulo Mineiro; UNIFESP: Universidade Federal de São Paulo; USP/SP: Universidade de São Paulo, Campus São Paulo; USP/RP: Universidade de São Paulo, Campus Ribeirão Preto.

3. Tratava-se de grupos que usaram o unitermo Fisioterapia-Terapia Ocupacional. 
Em uma distribuição por instituição, indicada no Gráfico 1, verifica-se uma concentração de Grupos de Pes- quisa na Universidade de São Paulo (USP) e na Universidade Federal de São Carlos (UFSCar), respectivamente.

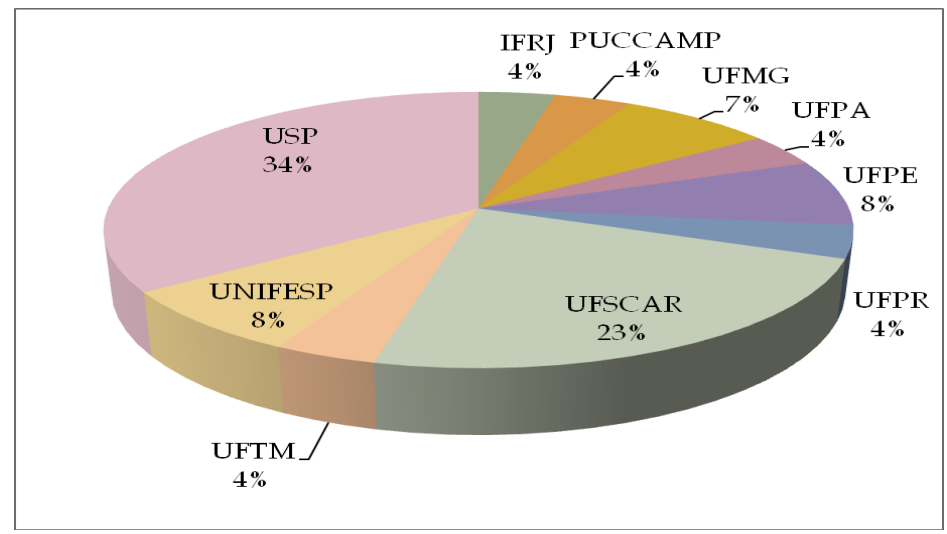

Fonte: $\mathrm{CNPq}(2010)$

IFRJ: Instituto Federal de Rio de Janeiro; PUC-Campinas: Pontifícia Universidade Católica de Campinas; UFMG: Universidade Federal de Minas Gerais; UFPA: Universidade Federal do Pará; UFPE: Universidade Federal de Pernambuco; UFPR: Universidade Federal do Paraná; UFSCar: Universidade Federal de São Carlos; UFTM: Universidade Federal do Triângulo Mineiro; UNIFESP: Universidade Federal de São Paulo; USP/SP: Universidade de São Paulo, Campus São Paulo; USP/RP: Universidade de São Paulo, Campus Ribeirão Preto.

Gráfico 1 - Distribuição de Grupos de Pesquisa, segundo Instituição de Ensino Superior

Regionalmente, conforme apresentado na Figura 1, observa-se uma expressiva concentração, $84 \%$ dos Grupos de Pesquisa estão na região Sudeste do Brasil, região que concentra, igualmente, os cursos de graduação e onde estão todos os pesquisadores vinculados a programas de pós-graduação. Esse retrato evidencia a necessidade de se ampliar o número de pesquisadores nas demais regiões para que se possa vislumbrar o crescimento científico da terapia ocupacional em todo o país. Essa situação reflete o pequeno número de doutores atuando na área que, segundo estimativas da Rede Nacional de Ensino em Terapia Ocupacional, aproxima-se de 120 pesquisadores, sendo que a maior parte deles, como se poderia antever, está na região Sudeste do Brasil.

A criação de Grupos de Pesquisa no Diretório do CNPq parece estar intimamente ligada à expansão do ensino de graduação em terapia ocupacional em Instituições de Ensino Superior, notadamente em universidades públicas. Observa-se que $50 \%$ dos atuais grupos surgiram entre os anos de 2008 e 2010, fato que pode estar vinculado ao
Programa REUNI (Reestruturação e Expansão das Universidades Federais) ${ }^{4}$, que criou, entre outros, cursos de graduação em terapia ocupacional em diferentes locais do país. O Gráfico 2 demonstra tal fato apresentando o número de grupos criados no período de 1989 a 2010.

Figura 1 - Distribuição de Grupos de Pesquisa, segundo Região do País

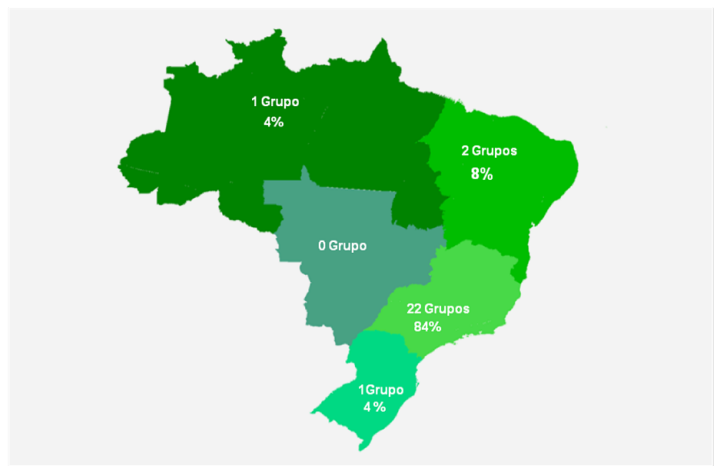

Fonte: CNPq (2010)

4. O Programa de Apoio a Planos de Reestruturação e Expansão das Universidades Federais Brasileiras é parte integrante de um conjunto de ações do Governo Federal no Plano de Desenvolvimento de Educação do MEC. Foi instituído pelo Decreto Presidencial № 6.096, de 24 de abril de 2007, com o objetivo de dar às instituições federais condições de expandir o acesso e garantir condições de permanência no Ensino Superior. Em síntese, suas diretrizes são: redução das taxas de evasão; ocupação das vagas ociosas e aumento do número de vagas ofertadas, principalmente no turno da noite. 


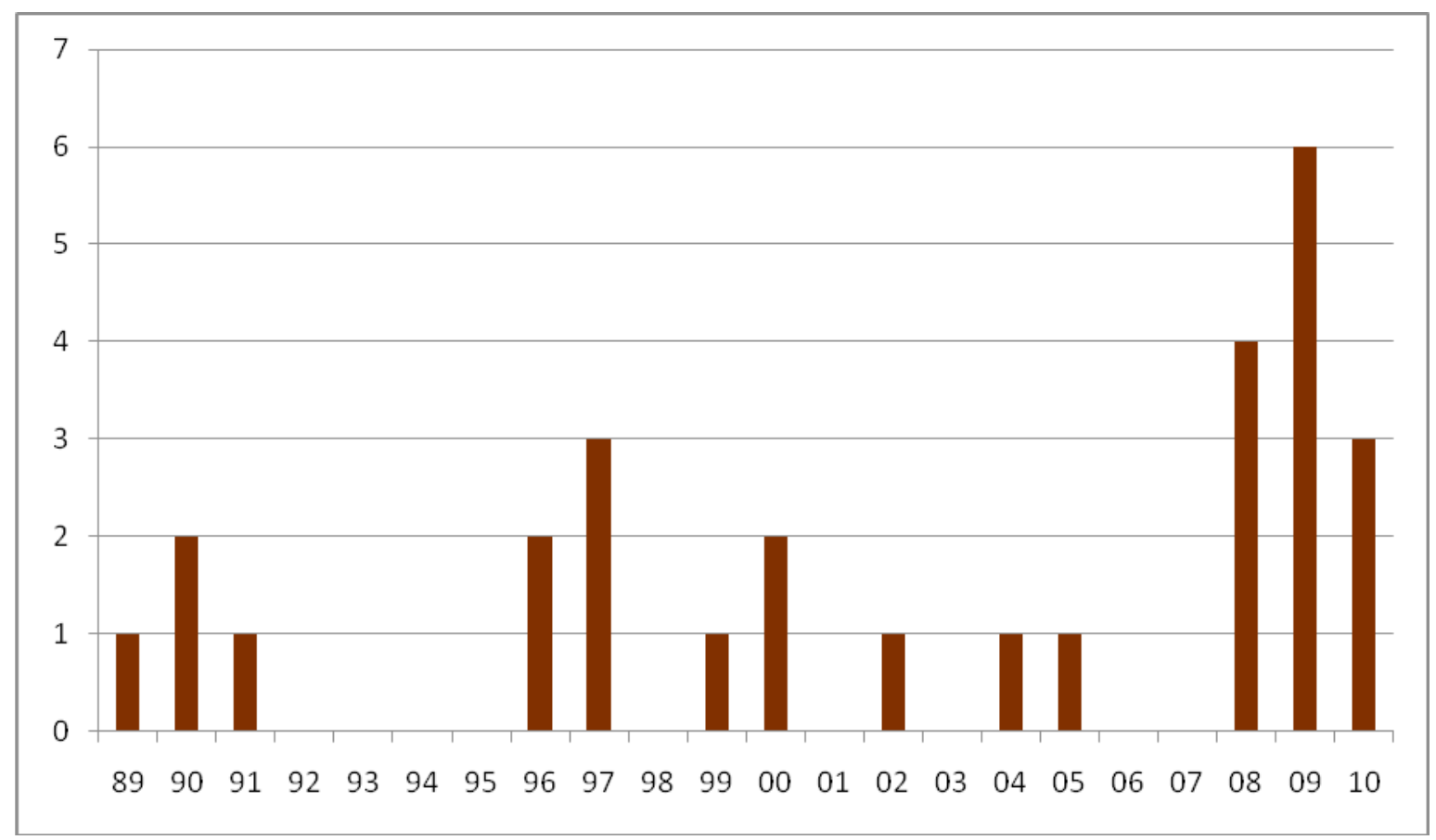

Fonte: CNPq (2010)

Gráfico 2 - Número de Grupos de Pesquisa em Terapia Ocupacional cadastrados no CNPq, segundo ano de criação

No contato realizado diretamente com os líderes dos 26 grupos considerados, foram solicitadas informações acerca dos pesquisadores e alunos integrantes, das pesquisas em desenvolvimento e dos financiamentos acessados.

Pôde-se constatar que os grupos de pesquisa na área de terapia ocupacional são compostos por 172 pesquisadores, dos quais 91 são doutores. Com relação aos 81 pesquisadores não-doutores, verificou-se que 16 têm doutorado em andamento, 41 são mestres, 6 estão com o mestrado em andamento, 10 são especialistas, 2 têm especialização em andamento e 6 são apenas graduados. São 30 os técnicos atuando nos diferentes grupos.

Constata-se que apenas $53 \%$ dos pesquisadores da área são doutores, demonstrando que a composição dos grupos de pesquisa se dá substancialmente por pessoas que não concluíram sua formação no nível de doutorado. Vale ainda destacar que há um grupo liderado por um mestre, o que é considerado um caso atípico pelo CNPq.

Segundo informações do Diretório de Grupos de Pesquisa do CNPq:

Pesquisadores são os membros graduados ou pósgraduados da equipe de pesquisa, direta e criativamente envolvidos com a realização de projetos e com a produção científica, tecnológica e artística do grupo. Se estiver matriculado em um curso de graduação ou pós-graduação (especialização, mestrado ou doutorado), deve ser incluido como estudante, desde que seu orientador seja um pesquisador do grupo. Estagiários pós-doutorais devem ser considerados como pesquisadores do grupo $(\mathrm{CNPq}$, 2010).

A partir dessa definição, apreende-se que há pessoas em formação que poderiam estar cadastradas como estudantes, ao invés de pesquisadores, se tivessem a possibilidade de realizar sua formação sob orientação de pesquisadores da área, fato que ocorre, mas de modo minoritário, em função do pequeno número de programas de pós-graduação.

Com relação aos estudantes cadastrados em tais grupos, obteve-e a informação de 186 estudantes, sendo: 117 estudantes com graduação em andamento, 25 estudantes com mestrado em andamento, 14 estudantes com doutorado em andamento e 30 estudantes em cursos de especialização ou outros.

Por meio desses dados, torna-se evidente um perfil de desenvolvimento da área via cursos de graduação, com uma concentração predominante de estudantes desse nível de formação, o que implica determinadas possibilidades de 
desenvolvimento de pesquisa, ou seja, propostas individuais e, quando sob orientação, aquelas do âmbito da iniciação científica.

Especificamente em relação aos projetos de pesquisa, foram informados 124 projetos em andamento, sendo que, segundo os líderes, 65 desses projetos são realizados com financiamento e, portanto, 59 não contam com financiamento. Entre os projetos financiados, 41 declararam receber verba de agência de fomento à pesquisa e 14 declararam, explicitamente, auxílio à pesquisa. Assim, infere-se que a maior parte do financiamento declarado relaciona-se ao acesso a bolsas de pesquisa, nos níveis de Iniciação Científica, Mestrado, Doutorado e Pós-Doutorado, sendo a menor parte composta por projetos que obtiveram recursos próprios para sua realização. Houve também informação de outras fontes de financiamento, como: prefeituras, ministérios e auxílio à atividade de extensão.

Foi possível observar, ainda, uma pulverização dos projetos elencados, em detrimento de um projeto temático central com subprojetos acoplados, levando à consideração de que cada projeto de iniciação científica, por exemplo, constitui-se, por si mesmo, em uma pesquisa independente.

Nas informações disponibilizadas pelo Diretório de Grupos de Pesquisa do CNPq fica explicitada a ideia de organicidade e desenvolvimento hierárquico de ações interligadas. O próprio conceito de grupo de pesquisa é claro:

trata-se de um grupo de pesquisadores, estudantes e pessoal de apoio técnico que está organizado em torno à execução de linhas de pesquisa segundo uma regra hierárquica fundada na experiência e na competência técnico-cientifica. Esse conjunto de pessoas utiliza, em comum, facilidades e instalações físicas. Como se vê, a(s) linha(s) de pesquisa subordina(m)-se ao grupo, e não o contrário. (...) Algumas vezes, inclusive, o que parece ser apenas uma linha de pesquisa, com pequenas variações, aparece como elemento organizador de vários grupos (CNPq, 2010).

Tem-se, portanto, um quadro que demonstra a necessidade de organização em torno da pesquisa em terapia ocupacional, por meio do delineamento de suas áreas e subáreas, do seu objeto de investigação, avançando para que se possa alcançar os financiamentos disponíveis e ingressar, formalmente e de forma fortalecida, no campo da produção de conhecimento científico brasileiro.

Por fim, no levantamento geral apresentado, destacamos as informações sobre o acesso a financiamentos da agência CNPq. O Edital Universal do CNPq, que objetiva: "selecionar propostas para apoio financeiro a projetos que visem contribuir significativamente para o desenvolvimento científico e tecnológico do país, em qualquer área do conhecimento" (CNPq, 2010), contemplou, em 2009, quatro propostas de pesquisadores terapeutas ocupacionais e, em 2010, três. É, evidentemente, um número pequeno, frente à necessidade de desenvolvimento da área. Porém, representantes da agência vêm apontando, constantemente, que a terapia ocupacional precisa incrementar substancialmente sua demanda para que possa ampliar o número de projetos aprovados. Isto tem sido difícil para os pesquisadores da área. No Edital Universal de 2009, apesar da proposta de 2008 de que todos os grupos de pesquisa submetessem pelo menos dois projetos de pesquisa, o número de solicitações da terapia ocupacional foi 18 (eram 25 os grupos de pesquisa no mesmo ano).

Ainda no âmbito do acesso ao financiamento de pesquisa no que tange ao $\mathrm{CNPq}$, a área dispõe de, apenas, quatro Bolsas de Produtividade em Pesquisa, demonstrando o precário reconhecimento de sua ação em pesquisa por esse órgão de fomento.

Esse cenário indica, por um lado, a necessidade de aprimoramento da área e, por outro, a necessidade de uma articulação e representatividade própria junto às agências de fomento para que os interesses da terapia ocupacional, considerados a partir de sua especificidade, possam ser apresentados, reconhecidos e lidados em ações que possibilitem seu crescimento e desenvolvimento.

\section{CONSIDERAÇÕES FINAIS}

Os diálogos e inserções da área de terapia ocupacional no âmbito da pesquisa não são fáceis e envolvem disputas acerca de suas regras, funcionamento e acessos junto a campos já consolidados de pesquisa e àqueles em constituição. Por exemplo, cada uma das subáreas do Comitê Multidisciplinar em Saúde, Educação Física, Fonoaudiologia, Fisioterapia e Terapia Ocupacional, podem ser consideradas, apesar de suas trajetórias distintas, como emergentes.

Nossa efetiva participação dependerá de nossa capacidade de organização coletiva para a ocupação dos espaços legitimados na CAPES, no CNPq e nos demais órgãos de fomento à pesquisa e à pós-graduação no país. Da mesma maneira, é necessária a ação conjunta para o desenvolvimento de pesquisas e conhecimentos que se apresentem como relevantes para a área e que se aglutinem num ponto comum de trabalho: a promoção da autonomia e da inclusão de grupos populacionais com os quais trabalhamos. Assim como já defendemos anteriormente:

Podemos inferir que, para a consolidação desse campo, muitas etapas deverão ainda ser cumpridas e enfrentadas. 
Certamente, tem-se hoje um contingente de pesquisadores, em diferentes momentos dessa carreira, que vêm acumulando experiência e condições para a realização das tarefas. Nosso pequeno número é um entrave, mas nossa qualidade tem sido, apesar disso, demonstrada. As perspectivas para a área como um todo dependerão de sua capacidade de avanço no âmbito da pesquisa, precisamos que isso reste claro em nosso horizonte profissional e que permaneçamos, como vimos fazendo, fortemente atuantes (LOPES; MALFITANO, 2009, p.120).

Está posta para a terapia ocupacional brasileira a possibilidade de crescimento no que concerne à pesquisa e à produção de conhecimento, sendo que, para tanto, será preciso enfrentar os desafios da qualificação de seus pesquisadores, do aumento do número de doutores, da criação de novos programas de pós-graduação stricto sen$s u$, de qualificação e reconhecimento dos seus periódicos específicos, do acesso efetivo a recursos para essa tarefa e, sobretudo, do tensionamento para que haja a valorização das análises de práticas sociais dedicadas à compreensão e à abordagem das temáticas com as quais se defrontam os terapeutas ocupacionais e que desafiam a área como campo de conhecimento e de intervenção.

LOPES, R. E.; MALFITANO, A. P. S.; OLIVER, F. C.; SFAIR, S. C.; MEDEIROS, T. J. Research in occupational therapy: notes about the academics ways in the national scene. Rev. Ter. Ocup. Univ. São Paulo, v. 21, n. 3, p. 207-214, set./dez. 2010.

\begin{abstract}
The area of occupational therapy has been discussing, in their collective forums, organizing strategies to access funding for research and its institutionalization in stricto sensu post-graduate studies. In this scenario, it's presented data on Research Groups in Occupational Therapy area in Brazilian universities. Groups registered with the term "Occupational Therapy" in their names, in the area of research title or in its keywords were searched in the CNPq Directory of Research Groups (large Health Area, Physical Therapy Sub-Area and Occupational Therapy). Subsequently, the groups' leaders were contacted and asked about data on ongoing researches, their participants as well as funds obtained. Twenty-six active research groups were found with great concentration in the southeast of Brazil and with a population of 172 researchers, from which 91 are doctors. One hundred twenty-four ongoing projects were i.dentified, 65 of which received some kind of financing. It is possible to verify that the area still has a small number of doctors with low participation in stricto sensu post-graduate programs, which are relevant elements for the development of specific research; it is necessary to deal with the challenge of achieving representative levels and grow along the institutions which certify the production of academic knowledge.
\end{abstract}

KEY WORDS: Research; Research financing; Directories of researchers; Occupational therapy/ trends; Education, higher.

\title{
REFERÊNCIAS
}

CNPq. Conselho Nacional de Desenvolvimento Científico e Tecnológico. Diretório de Grupos de Pesquisa [Citado em 22 set. 2010]. Disponível em: http://dgp.cnpq.br/buscaoperacional/.

LOPES, R. E., OLIVER, F. C.; MALTITANO, A. P. S.; GALHEIGO, S. M.; ALMEIDA, M. C. XI Encontro Nacional de Docentes de Terapia Ocupacional: refletindo sobre os processos de formação acadêmica e profissional. Rev. Ter. Ocup. Univ. São Paulo, v. 19, n. 3, p. 159-166, 2008.

LOPES, R. E.; MALFITANO, A. P. S.; OLIVER, F. C.; HAHN, M. S. I Seminário Nacional de Pesquisa em Terapia Ocupacional: perspectivas e consolidação do Campo. Cad. Ter. Ocup. UFSCar, v. 17, Supl. Esp., p. 7-10, 2009.

LOPES, R. E.; MALFITANO, A. P. S. Perspectivas e desafios para a pesquisa em terapia ocupacional: uma análise do seu I Seminário Nacional de Pesquisa. Cad. Ter. Ocup. UFSCar, v. 17, Supl. Esp., p. 115-120, 2009.

OLIVER, F. C. Disseminação do conhecimento e periódicos científicos: contribuições para o debate em terapia ocupacional. Cad. Ter. Ocup. UFSCar, v. 17, Supl. Esp., p. 69-85, 2009. 